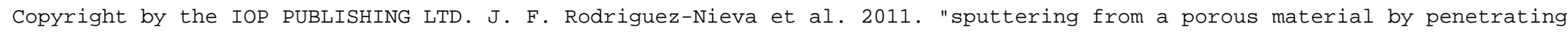
ions," ApJ 743 L5 doi:10.1088/2041-8205/743/1/L5

\title{
SPUTTERING FROM A POROUS MATERIAL BY PENETRATING IONS
}

\author{
J. F. Rodriguez-Nieva ${ }^{1}$, E. M. Bringa ${ }^{2}$, T. A. Cassidy ${ }^{3}$, R. E. Johnson ${ }^{4}$, A. Caro ${ }^{5}$, M. Fama ${ }^{4}$, \\ M. J. LOEFFLER ${ }^{6}$, R. A. BARAGIOLA ${ }^{4}$, AND D. FARKAS ${ }^{7}$ \\ ${ }^{1}$ Instituto Balseiro, Universidad Nacional de Cuyo, 8400 Bariloche, Argentina \\ ${ }^{2}$ CONICET \& Instituto de Ciencias Básicas, Universidad Nacional de Cuyo, 5500 Mendoza, Argentina \\ ${ }^{3}$ CalTech/JPL, Pasadena, CA 91109, USA \\ ${ }^{4}$ Laboratory for Atomic and Surface Physics, University of Virginia, Charlottesville, VA 22903, USA \\ ${ }^{5}$ Los Alamos National Laboratory, Los Alamos, NM 94551, USA \\ ${ }^{6}$ NASA Goddard Space Flight Center, Astrochemistry Branch, Code 691, Greenbelt, MD 20771, USA \\ ${ }^{7}$ Department of Materials Sciences, Virginia Tech, Blacksburg, VA 24061, USA \\ Received 2011 August 25; accepted 2011 October 11; published 2011 November 15
}

\begin{abstract}
Porous materials are ubiquitous in the universe and weathering of porous surfaces plays an important role in the evolution of planetary and interstellar materials. Sputtering of porous solids in particular can influence atmosphere formation, surface reflectivity, and the production of the ambient gas around materials in space. Several previous studies and models have shown a large reduction in the sputtering of a porous solid compared to the sputtering of the non-porous solid. Using molecular dynamics simulations we study the sputtering of a nanoporous solid with $55 \%$ of the solid density. We calculate the electronic sputtering induced by a fast, penetrating ion, using a thermal spike representation of the deposited energy. We find that sputtering for this porous solid is, surprisingly, the same as that for a full-density solid, even though the sticking coefficient is high.
\end{abstract}

Key words: atomic processes - methods: numerical - molecular processes - planets and satellites: surfaces radiation mechanisms: general

Online-only material: color figure

\section{INTRODUCTION}

Porous materials play an important role in the evolution and structure of interstellar and interplanetary environments. The effect of porosity has been considered when calculating optical properties (Shen et al. 2008), collisions (Ormel et al. 2009), and chemistry (Perets \& Biham 2006), but its influence on desorption has not been considered in depth. Most experimental work on sputtering (Baragiola 2004) focuses on full-density materials and assumes that simple models (Johnson 1989) to extrapolate sputtering yield values from these compact solids to values for porous solids. There are recent efforts to change this outlook. Early experiments simulated a porous regolith (Hapke \& Cassidy 1978; Hapke 1986, 2001), and recent experiments studied porous silicates that simulate regoliths found in the solar system (Loeffler et al. 2009). These experiments suggested that there is a significant decrease in the sputtering yield as compared to the full-density sample, explained as re-deposition of sputtered atoms on neighboring grain walls. They are also consistent with Monte Carlo (MC) modeling (Cassidy \& Johnson 2005), which showed that the sputtering yield for porous materials was significantly lower than in non-porous ones when the sticking coefficient of ejected atoms was large. They found that the decrease in yield is mostly associated with re-deposition of atoms ejected from internal surfaces of the sample.

Current models and experiments-which have concentrated on large-scale porosity $(10-500 \mu \mathrm{m})$-could benefit from simulations at the atomic level. Molecular dynamic (MD) simulations follow the evolution of a system of atoms interacting through an effective potential, integrating their equations of motion, and have been used to study electronically induced sputtering (Bringa \& Johnson 2002). MC methods such as SRIM (Ziegler 2003) offer flexibility and speed in calculating sputtering yields, but typically cannot account for nonlinear effects in the nuclear stopping regime, and would give zero sputtering yields in the electronic sputtering regime, since a link between electronic energy deposition and lattice atom motion is not provided. MD, on the other hand, can reproduce experimental results for simple solids, such as pure metals and semiconductors, and it can include chemical sputtering and electronic effects through different models (Bringa \& Johnson 2002). Recently, plasma etching from a surface of $\mathrm{SiOCH}$ with nanometer-sized pores bombarded by $300 \mathrm{eV} \mathrm{CF} 2$ ions was studied by Smirnov and co-workers using MD (Smirnov et al. 2007). At normal incidence, the sputtering yield for the porous sample (65\% density) was $\sim 62.5 \%$ of the yield for the full-density sample.

Nano-porous materials, such as water ice (Palumbo 2006; Raut et al. 2007), are present throughout astronomical environments, and thus here we present MD simulation results of the sputtering from a nano-porous solid (pore size of 2-30 nm) caused by a fast ion that produces a damage track along its path through the material. First we describe simulation details, then we present the sputtering yield results and finally we discuss these results and their possible application.

\section{SIMULATION DETAILS}

MD runs were carried out using the MD software LAMMPS ${ }^{8}$ (Plimpton 1995). Our simulations rely on the same principles used in previous simulations for compact targets (Bringa \& Johnson 2002; Mookerjee et al. 2008). We use a Lennard-Jones (LJ) potential to describe the interaction between simulated particles, which can represent either atoms or whole molecules. Sputtering of molecular solids shows that the majority of ejecta are non-dissociated molecules (Baragiola 2004) and,

\footnotetext{
8 http://lammps.sandia.gov
} 
therefore, we can refer to our particles as molecules in the case of molecular targets. Simulations including an atomistic representation of molecules (Bringa \& Johnson 2000), oxides (Devanatham et al. 2007), etc., are possible but much more computationally expensive and also display similar behavior for sputtering from a thermal spike. The LJ potential has two parameters: length $(\sigma)$ and energy $(\varepsilon)$. Typically, one can fit these numbers by using the density $\rho=1.055 \sigma^{-3}$ and cohesive energy $U \sim 8 \varepsilon$ of the solid being studied. Simulations are dimensionless and, therefore, a given material can be chosen a posteriori to compare to laboratory data as needed (Bringa \& Johnson 2004; Bringa et al. 2007). For instance, for water ice, $\rho \sim 30 \mathrm{~nm}^{-3}$ and $U \sim 0.5 \mathrm{eV}$. Because of this, temperature has units of $\varepsilon / k_{\mathrm{B}}$, time $\left(m \sigma^{2} / \varepsilon\right)^{1 / 2}$, pressure $\varepsilon / \sigma^{3}$, etc.

\subsection{Sample Generation}

The main purpose of this paper is to understand the role of nanoscale porosity on sputtering, without necessarily reproducing the fine details of porous structures in space, which are very complicated and probably cannot be completely generalized. Most experimental studies deal with porous materials formed by large "fused" grains. Here we describe materials on a molecule-by-molecule basis. The simulated nanoscale solids are made of "filaments," composed of hundreds of molecules, which interlock with each other.

The porous samples were generated as in Crowson et al. (2009). Radiation damage in the nuclear stopping regime was recently studied in similar bulk samples (Bringa et al. 2011). For our simulations we chose samples that were of $55 \%$ of the solid density ( $45 \%$ porosity), had a distribution of ligament/grain sizes around 5-8 $\sigma$, and were composed of $\sim 195,000$ particles. For comparison with our porous sample, we also used a compact sample ( $0 \%$ porosity), which was a pure single crystal with $\sim 352,500$ particles. The sample sizes are generally limited to computational constraints, as MD of ion bombardment cannot simulate samples with more than few million atoms, yet our samples were large enough that the boundaries did not affect the sputtering results. In order to obtain reliable sputtering yields, $10^{2}$ to $10^{4}$ events are needed to accumulate statistics, which leads to a severe limitation in the size of the pores in simulated samples.

For convenience we used approximately cubic samples, with a side of $\sim 70 \sigma$. All samples were run with periodicity in the $x y$-plane to simulate "infinite" surfaces, while free surfaces were used in the $z$-direction as described in earlier work (Bringa \& Johnson 2002). The outermost regions of thickness $2 \sigma$ in the $x y$-directions were kept fixed, and a region inside that, of width $2 \sigma$, was thermostated, i.e., kept at constant temperature $T=$ 0.1 , using an overdamped Langevin thermostat. Such boundary conditions in the $x y$-directions help quenching possible shock waves produced by the ion tracks at high excitation densities, while cooling the sample at long times. Prior to depositing the energy, samples were relaxed with a thermostat at $T=0.1$ in 1500 steps to allow for some surface reconstruction.

A schematic of the porous and compact sample is shown in Figure 1; interestingly, we note that our sample also looks qualitatively similar to a sample with "fused" grains (Loeffler et al. 2008), even though the scale of porosity is orders of magnitude different.

\subsection{Track Evolution and Sputtering Yield}

In this work, we focus on the role of ion tracks in sputtering caused by fast ions with energies $>\sim 10 \mathrm{keV} \mathrm{amu}^{-1}$. Sputtering induced by these ions is important in a number of planetary and interstellar environments, such as Europa. In our simulations, we assumed that the ions primarily deposited their energy in a track cylinder of radius $2 \sigma$, which is typical for a number of astrophysical and solar system conditions (Bringa \& Johnson 2004). That is, after initially cooling and relaxing the sample, all molecules within the track cylinder were given a Gaussian velocity distribution producing an increased temperature of track molecules. Following a scenario given by the two-temperature model (Mookerjee et al. 2008), the temperature of the molecules inside the track was ramped up during a number of steps, to reach an average temperature of $T=10$ (in LJ units).

We have focused here on ions incident along the surface normal. We neglect possible changes in track parameters as it traverses the sample. We note that the filaments in our porous sample are small: a single ion track can include regions from several filaments. The net energy deposited was less than that in the full-density case, by a factor of $\sim\left(\rho / \rho_{0}\right)$ because the track volume would always include voids. This is significantly different from the assumptions by Cassidy \& Johnson (2005) in which the grains were assumed to be large, the porosity of interest was the volume between these large grains, and the ion only affected the "surface" layers of individual grains. We used samples that were large enough along $z$ that sputtering from the top surface was not influenced by the bottom surface (see Figure 2), and therefore a single run produces two estimates of the sputtering yield. In order to calculate the sputtering yield, we counted all molecules having a $z$-coordinate $7.5 \sigma$ away from the surface. For each simulation, we always used the pristine sample but the position of the track was changed, displacing it around the center of the box up to $\sim 4 \sigma$ to obtain a statistically meaningful number of ejected molecules. It was enough to do 50 simulations (100 sputtering events) to give an error in the yield within a range of 3\%-10\%. Figure 2 shows a typical slice of the samples during the simulation.

\section{ANALYSIS OF SPUTTERING RESULTS}

Most of the ejection occurs promptly due to energetic molecules near the surface region, with a smaller contribution at much later times from the sub-surface region. Therefore, most simulations were followed during nearly $2 \times 10^{4}$ steps to include the latter contribution. At the end of the simulations, the sample temperature had recovered to the starting temperature ( $T \sim 0.1)$ for both the porous and the full-density solid.

Figure 3 shows the energy, depth of origin, and radius of origin from the track center for the ejected particles. Sputtering of the full-density solid only comes from molecules initially located in the first and second layers, and from inside the track. Sputtering in the nanoporous material, on the other hand, has important contributions from up to the sixth layer with small contributions from as far down as the 25 th layer and also from large radii.

The sputtering yields (molecules ejected per ion) for both the porous and full-density samples are similar for prompt energy deposition $Y(55 \%)=6.3 \pm 0.3$ and $Y(100 \%)=6.5$ \pm 0.4 , in agreement with previous modeling for dense solids (Bringa \& Johnson 2004). Interestingly our results contrast with those found in previous studies on sputtering of materials porous on the micron scale, which show large reductions in both experiments (Loeffler et al. 2009 shows $\sim 70 \%$ reduction at intermediate fluence) and MC simulations (Cassidy \& Johnson 2005 show $75 \%$ reduction when the sticking coefficient is 1 ). In the MC simulations, the ion penetration depth was much smaller 


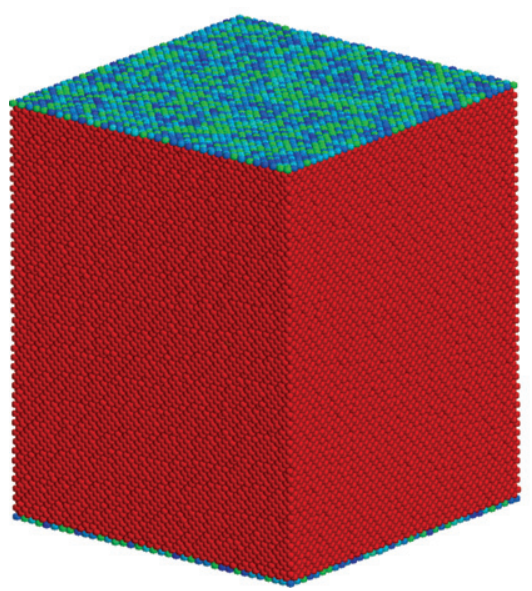

(a)

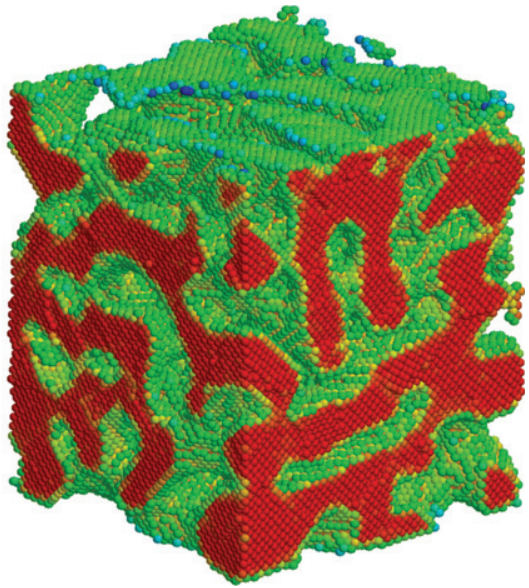

(b)

Figure 1. Initial samples: full density (left) and porous (right), showing bulk molecules in red and surface molecules in blue (100\% density) or green/blue (55\% density).

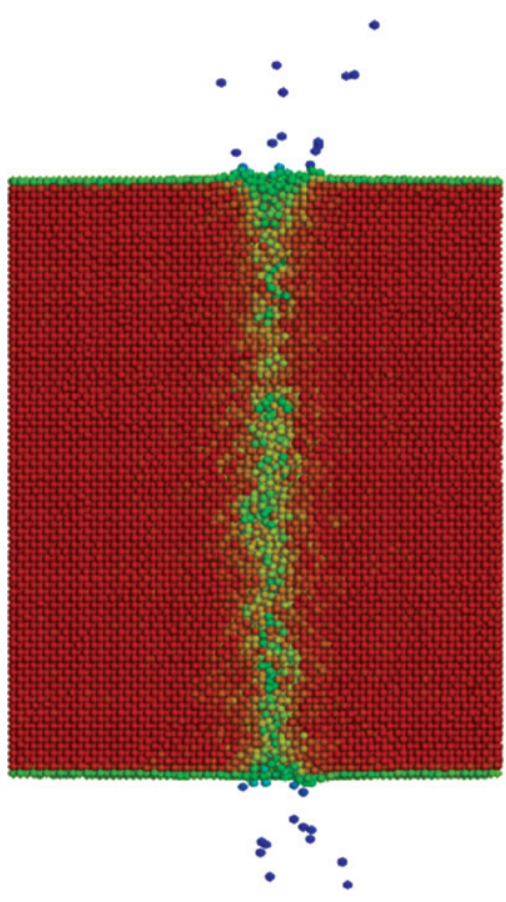

(a)

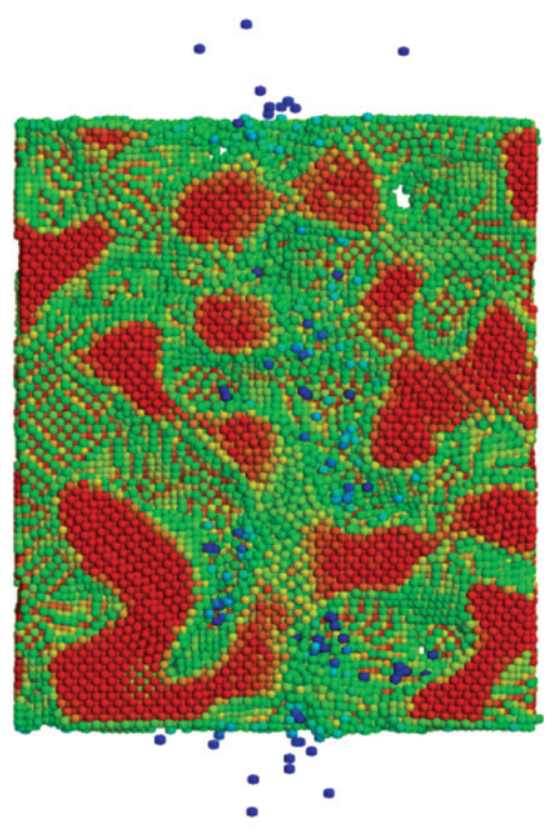

(b)

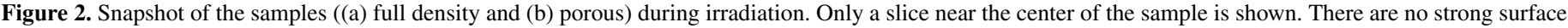

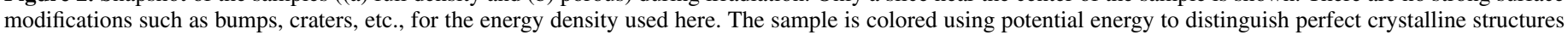
(red) from defects (green and yellow) and ejected molecules (blue).

than the grain size and thus introducing porosity mostly served to increase the number of internal regions where the ejected atoms could redeposit. In our simulations, the ions penetrate entirely through the solid and thus porosity causes less energy to be deposited, which would contribute to a decrease in sputtering. On the other hand, the morphology of the surfaces is such that the actual physical surface due to roughness is larger than the cross-section surface area, enhancing the sputtering. These two opposite effects compensate each other in our simulations, giving a similar final yield. Our electronic sputtering case is somewhat akin to the nuclear sputtering case, which does not depend on the atomic density of the target, provided that the binding energy stays the same (Sigmund 1969). This is because the energy deposition is directly proportional to the density, but the escape depth increases inversely proportional to the density.
A similar increase in escape depth is seen in the case under study here (Figure 3).

In addition, there are other second-order effects: the foam remains hot over times longer than the bulk sample, contributing slightly more to low-energy quasi-thermal ejection. In a few cases we observed ejection of small clusters. The qualitative foam topology was not greatly affected by a single ion track, and we do not observe any compaction of the samples within our simulated time. However, we do not consider the case of cumulative bombardment, which could be crucial for compaction.

As in the Cassidy simulations, visualization of our simulations indicates that the atoms ejected from surfaces of the porous sample stick to opposite surfaces or escape if they leave a pore leading to a surface opening. We cannot calculate directly 


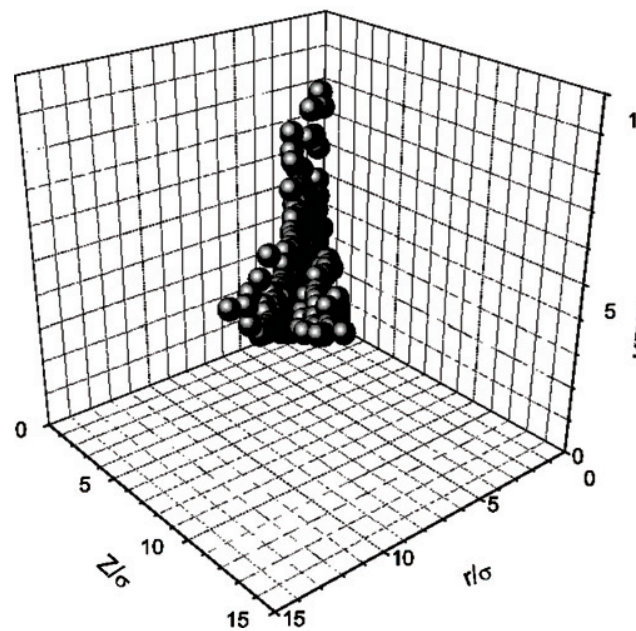

(a)

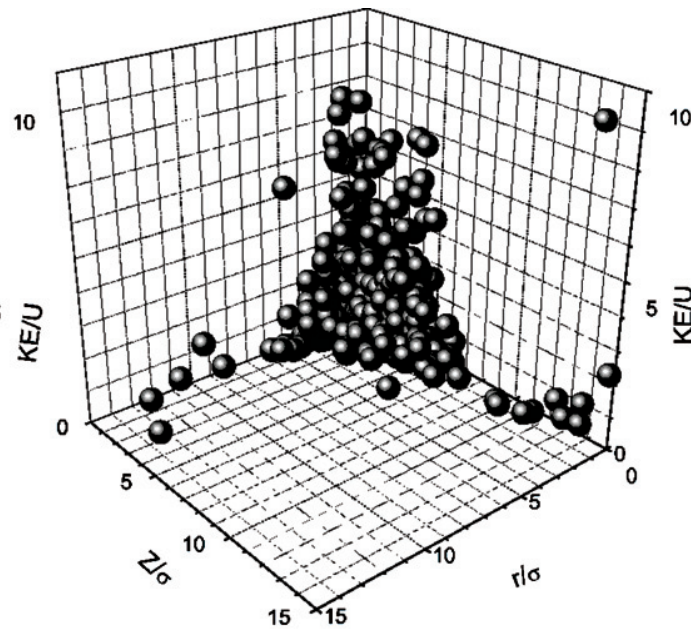

(b)

Figure 3. Kinetic energy (normalized to the binding energy $U$ ), depth, and radius of origin of ejected particles. Full density (left) and porous sample (right). As expected, there are longer "tails" for the nanoporous material regarding the depth and radius of origin.

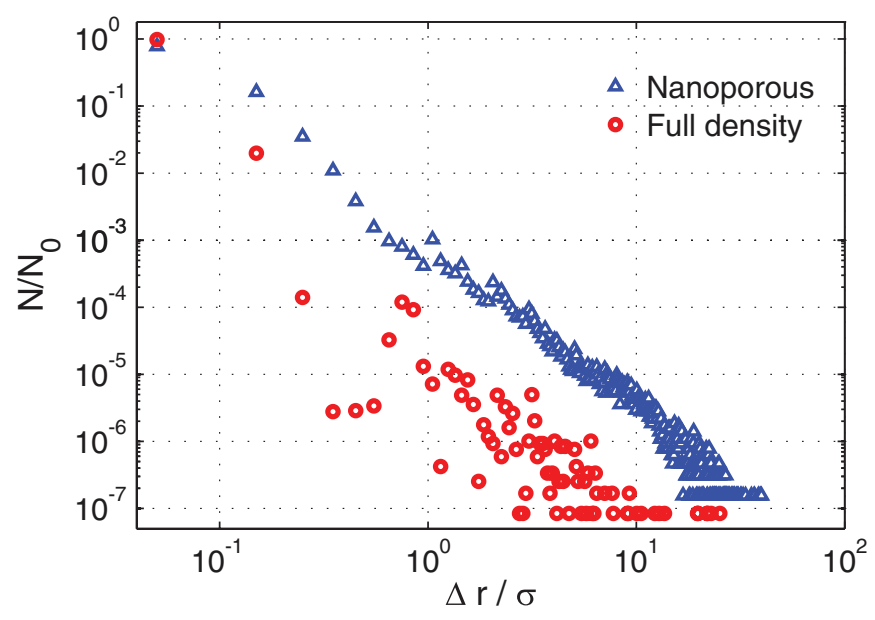

Figure 4. Distribution of displacements. Here $\Delta r$ represents the net displacement of atoms between the start and finish of the simulation. Atoms displaced more than $\Delta r \sim 10 \sigma$ are considered sputtered from inner surfaces and re-deposited. Sputtered atoms are excluded from this analysis.

(A color version of this figure is available in the online journal.)

the sticking coefficient in our simulations. However, we can indirectly calculate it by following the atoms ejected from internal surfaces and determining what percentage remains in the sample. To obtain this, we have analyzed the distribution of atomic displacements with respect to their original positions. In Figure 4, we show these displacements for all atoms that remain in the target. There is melting and great mobility of atoms inside the track, but this motion rarely involves displacements larger than a few times the radius of the track. Therefore, we can assume that an atom that travels more than the size of the filaments $(5 \sigma-8 \sigma)$ has been ejected from a surface and crossed a void inside the porous sample. Since the atoms counted here all have low energy and exclude sputtered atoms, those ejected atoms that crossed a void had to be re-deposited in another surface, without the possibility of multiple scattering. Two extreme cases can be considered: (a) atoms within the ejection depth of Figure 3 (atoms located within $\sim 15 \sigma$ of the surface), and (b) atoms inside a layer of the same thickness, but located far from the surface. In both cases, $\sim 1.7 \%$ of the atoms are ejected, which is consistent with the uniform energy deposition in our simulations. Out of the total number of "ejected" atoms from the surface layer (i.e., those that traveled a distance $\Delta r>$ 10 but remain in the sample and those that were ejected from the sample and contribute to the sputtering yield), only $44 \%$ of the atoms are re-deposited, while the rest escape without colliding with any surface. For the deeper layer $100 \%$ re-deposit, indicating that the effective atomic sticking is indeed one in our simulations.

\section{CONCLUSIONS}

In this paper, we model a simple, one component porous solid of interest to the understanding of the sputtering of the porous surface layer in many astrophysical environments. Future advances in computing and modeling will allow us to tackle more realistic porosities and compositions, but it is clear that nanoscale porosity differs significantly from micron-size porosity. To model sputtering yields one needs at least two target parameters: its porosity and its filament/grain size compared to the ion track or collision cascade. Sputtering at the nanoscale is dominated by ejection of atoms from the enhanced surface area near the top surface. If one takes into account void sizes and grain sizes of 10-1000 $\mu \mathrm{m}$ size, the scenario will be as described by Cassidy \& Johnson (2005), where ions deposit their energy in a single grain, possibly deep below the surface. This differs from the density-depleted sample we have, where width and extent of a single ion track overlaps several "grains," and where pores and grains are much smaller than the penetration depth of the ion. Our results for pores at the nanoscale could then be applied, for instance, to ice water surfaces, where the pore size is nanoscopic. In addition, there might be cases where large grains in regolith or meteorites could be carpeted by much smaller nanometer-scale grains, as sometimes observed (Sears \& Kral 1998). We find that the sputtering is about the same for the porous and the bulk sample and we do not find compaction of our samples for a single ion track, but cumulative bombardment might lead to different results.

In summary, if the incident ion can sputter from several grains, the reduction/enhancement in sputtering will depend on porous topology (void size and distribution) in the region of the surface up to a depth of couple of ion ranges, similarly to what occurs in a rough surface. 
Further simulation work is needed for off-normal ion incidence, details on ejected ions, dependence on track radii, etc. The thermal spike model used here can give quantitative sputtering results, comparable to experiments, only after a careful choice of parameters (Bringa \& Johnson 2000, 2004). Experiments using sub-micron/micron powders (silica, alumina) bombarded by heavy ion of hundreds of keVs might be able to test our results. Another possibility is to measure kilo-electron volt sputtering from porous carbon or metallic foams, where electronic sputtering might be negligible.

E.M.B. thanks support from CONICET and grant PICT2009-0092. J.F.R.N. thanks support from the Comisión Nacional de Energía Atómica (CNEA) for a scholarship. R.A.B. thanks NSF Astronomy for financial support.

\section{REFERENCES}

Baragiola, R. A. 2004, Phil. Trans. R. Soc. A, 362, 29

Bringa, E. M., \& Johnson, R. E. 2000, Surf. Sci., 451, 108

Bringa, E. M., \& Johnson, R. E. 2002, Phys. Rev. Lett., 88, 165501

Bringa, E. M., \& Johnson, R. E. 2004, ApJ, 603, 159

Bringa, E. M., Monk, J. D., \& Caro, A. 2011, Nanoletters, in press

Bringa, E. M., Kucheyev, S. O., Loeffler, M. J., et al. 2007, ApJ, 662, 372
Cassidy, T. A.. \& Johnson, R. E. 2005, Icarus, 176, 499

Crowson, D. A., Farkas, D., \& Corcoran, S. G. 2009, Scr. Mater., 61, 497

Devanatham, R., Durham, P., Du, J., Corrales, L. R., \& Bringa, E. M. 2007, Nucl. Instrum. Methods Phys. Res. B, 255, 172

Hapke, B. 1986, Icarus, 66, 270

Hapke, B. 2001, J. Geophys. Res., 106, 10

Hapke, B., \& Cassidy, W. 1978, Geophys. Res. Lett., 5, 297

Johnson, R. E. 1989, Icarus, 78, 206

Loeffler, M. J., Baragiola, R. A., \& Murayama, M. 2008, Icarus, 196, 285

Loeffler, M. J., Dukes, C. A., \& Baragiola, R. A. 2009, J. Geophys. Res., 114 E03003

Mookerjee, S., Beuve, M., Khan, S. A., Toulemonde, M., \& Roy, A. 2008, Phys. Rev. B, 78, 045435

Ormel, C. W., Paszun, D., Dominik, C., \& Tielens, A. G. G. M 2009, A\&A, 502,845

Palumbo, M. E. 2006, A\&A, 453, 903

Perets, H. B., \& Biham, O. 2006, MNRAS, 365, 801

Plimpton, S. J. 1995, J. Comput. Phys., 117, 1

Raut, U., Teolis, B. D., Loeffler, M. J., et al. 2007, J. Chem. Phys., 126, 244511

Sears, D. W. G., \& Kral, T. A. 1998, in LPI XXIX, SEM Imaging of Martian and Lunar Meteorites and Implications for Microfossils in Martian Meteorites, abstract No. 1934

Shen, Y., Draine, B. T., \& Johnson, E. T. 2008, ApJ, 689, 260

Sigmund, P. 1969, Phys. Rev., 184, 383

Smirnov, V. V., Stengach, A. V., Gaynullin, K. G., Pavlovsky, V. A., Rauf, S., \& Ventzek, P. L. G. 2007, J. Appl. Phys., 101, 053307

Ziegler, J. F. 2003, SRIM 2003.26 (www.srim.org) 